\title{
Síndrome lipodistrófica do HIV e seus fatores associados: um estudo em um hospital universitário
}

\author{
Lipodystrophic syndrome of HIV and associated factors: \\ a study in a university hospital
}

Lídia Laís Gomes Silva (http://orcid.org/0000-0001-7673-7680) ${ }^{1}$

Eryka Maria dos Santos (https://orcid.org/0000-0002-0317-7456) ${ }^{1}$

Luciana Caroline Paulino do Nascimento (https://orcid.org/0000-0002-7447-9670) ${ }^{1}$

Mikaella Carla de França Cavalcanti (https://orcid.org/0000-0003-2426-1582) ${ }^{1}$

Ilma Kruze Grande de Arruda (https://orcid.org/0000-0002-7142-1967) ${ }^{1}$

Marcella Campos Lima Luz (https://orcid.org/0000-0001-9226-1387) ${ }^{1}$

Poliana Coelho Cabral (https://orcid.org/0000-0002-2709-4823) ${ }^{1}$

${ }^{1}$ Programa de Residência em Nutrição Clínica, Hospital das Clínicas, Universidade Federal de Pernambuco. Av. Prof

Moraes Rego 1235, Cidade Universitária. 50670-

901 Recife PE Brasil. lidialaisgs@gmail.com

\begin{abstract}
The use of antiretroviral drugs has increased the survival of HIV patients, but may have side effects, such as lipodystrophic syndrome. This article aims to identify the frequency of the lipodystrophic syndrome and its associated factors in patients with HIV using antiretroviral therapy. It involved a cross-sectional study with HIV patients, monitored on an outpatient basis. The syndrome was evaluated by the association of two parameters: peripheral weight loss through the lipodystrophy severity scale and central fat accumulation, measured by the hip waist ratio. Poisson regression analysis was performed to identify the associated variables. Of the 104 patients evaluated, $27.9 \%$ presented the syndrome. After adjustment, the female sex $\left(P R_{\text {adiusted }}=2.16 C I_{950}\right.$

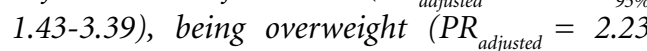
$C I_{95 \%}$ 1.35-2.65) and a longer period of use of antiretrovirals $\left(P R_{\text {adiusted }}=1.64 C I_{95 \%} 1.16-2.78\right)$, remained positively associated with the syndrome. On the other hand, a negative association with $C D 4$ count $£ 350\left(P R_{\text {adiusted }}=0.39 C I_{95 \%} 0.10-0.97\right)$ was observed The high prevalence of the syndrome and its association with specific groups reinforce the need for adequate follow-up and early identification to intervene in modifiable factors.

Key words Lipodystrophy, AIDS, Anthropometry, Antiretroviral therapy
\end{abstract}

Resumo $O$ uso de antirretroviral aumentou a sobrevida dos portadores do HIV, porém pode acarretar efeitos colaterais, como a sindrome lipodistrófica. O objetivo deste artigo é identificar a frequência da sindrome lipodistrófica e seus fatores associados em pacientes portadores do HIV em uso de terapia antiretroviral. Estudo transversal com pacientes acompanhados ambulatorialmente. A sindrome foi avaliada pela associação de dois parâmetros: emagrecimento periférico através da escala de gravidade de lipodistrofia e acúmulo de gordura central, mensurado pela relação cintura quadril. Para identificar as variáveis associadas foi realizada a análise de Regressão de Poisson. Dos 104 pacientes avaliados, 27,9\% apresentaram a síndrome. Após ajuste, ser do sexo feminino $\left(R P_{\text {ajustada }}=2,16 I C_{95 \%} 1,43-3,39\right)$, ter excesso de peso $\left(R P_{\text {aiustada }}=2,23 I_{95 \%} 1,35-2,65\right)$ e um maior tempo de uso dos antirretrovirais $\left(R P_{\text {ajustada }}=1,64\right.$ $\left.I C_{95 \%} 1,16-2,78\right)$ permaneceram positivamente associados à sindrome. Por outro lado, foi observada uma associação negativa com a contagem de CD4 $\mathfrak{E} 350\left(R P_{\text {ajustada }}=0,39 I C_{95 \% \%} 0,10-0,97\right)$. A alta prevalência da sinndrome e sua associação com grupos especificos reforçam a necessidade do adequado acompanhamento e identificação precoce como forma de intervir nos fatores modificáveis.

Palavras-chave Lipodistrofia, AIDS, Antropometria, Terapia antirretroviral 


\section{Introdução}

O tratamento para a infecção pelo vírus da imunodeficiência humana (HIV) inclui a terapia antirretroviral (TARV), a qual age inibindo a replicação do vírus, e representa um marco na terapêutica da síndrome da imunodeficiência adquirida (AIDS) e no melhor prognóstico, pois aumentou tempo de sobrevida desses pacientes ${ }^{1}$. O Brasil tem importante atuação na disponibilização desses medicamentos pelo Sistema Único de Saúde (SUS), crescendo anualmente o número total de pessoas vivendo com HIV (PVHIV) em tratamento, sendo observado um aumento de $53,2 \%$ nos casos no período de 2009 a $2014^{2}$.

No entanto, uma grande preocupação da Organização Mundial da Saúde (OMS) é a condição de vida desses pacientes, uma vez que apesar de maior tempo de sobrevida, o uso da TARV acarreta inúmeros efeitos colaterais, entre eles a síndrome lipodistrofica do HIV (SLHIV), conhecida simplesmente por lipodistrofia (LD). Este desfecho está associado a um maior risco cardiovascular, morbidade, mortalidade além de modificar a estética corporal, comprometendo a autoestima e levando a uma redução na taxa de adesão à TARV ${ }^{3,4}$.

A SLHIV foi inicialmente descrita como uma combinação de perda de gordura nos membros (lipoatrofia) e acúmulo de gordura central (lipohipertrofia), sendo nomeada pela primeira vez como síndrome em 1998, e incluiu além das alterações corporais, os distúrbios metabólicos ${ }^{5,6}$.

A prevalência de SLHIV varia entre $10 \%$ e $80 \%$. Essa grande variação pode estar relacionada a fatores geográficos, etários, genéticos e de estilo de vida, bem como a diferenças metodológicas usadas para o diagnóstico entre os estudos ${ }^{7-10}$.

Atualmente, não existe nenhum consenso quanto ao método diagnóstico da SLHIV, sendo várias as abordagens metodológicas ${ }^{11-14}$. A aplicação de questionários que utilizem o autorrelato em concordância com avaliador pode ser indicador precoce de mudanças corporais. Após essa avaliação clínica, geralmente a antropometria é associada para facilitar a identificação da alteração de gordura e posterior diagnóstico da SLHIV $^{15,16}$.

Embora não exista um parâmetro confiável que reflita alterações na distribuição da gordura específicas à SLHIV, alguns autores têm utilizado a relação cintura/quadril (RCQ $)^{17}$, pois correlaciona-se com a gordura total do abdômen, quando avaliada por TC, tendo como vantagem não requerer aparelhos sofisticados ${ }^{18}$.
A patogênese da SLHIV em PVHIV é multifatorial e ainda não está totalmente elucidada. Apesar de ter sido relacionada primeiramente ao tipo e ao tempo de TARV, outros fatores vêm sendo investigados, como a própria infecção pelo HIV, fatores genéticos, o estilo de vida do indivíduo, a idade e a gravidade dos marcadores de doença, a contagem de linfócitos T-CD4+ (LT-CD4+) e a carga viral. Esses fatores podem conduzir a alterações metabólicas como dislipidemias e mudanças no metabolismo da glicose, resultando em complicações cardiovasculares ${ }^{17,19-22}$.

Sendo assim, torna-se relevante identificar a síndrome e sua frequência e a partir de então intervir nos fatores associados modificáveis, para um melhor manejo das implicações à saúde das PVHI em uso da TARV. O objetivo deste trabalho foi identificar a frequência da SLHIV e seus fatores associados em PVHIV em uso de antirretrovirais.

\section{Métodos}

Foi realizado um estudo do tipo transversal, envolvendo PVHIV atendidos no ambulatório de doenças infecto parasitárias do Hospital das Clínicas da Universidade Federal de Pernambuco, no período de março a julho de 2017. A amostra abrangeu todos os pacientes que se enquadrarem nos critérios de elegibilidade da pesquisa durante o período de estudo. Foram inclusos aqueles pacientes em uso de TARV a pelo menos 6 meses, de ambos os sexos e com faixa etária $\geq 20$ anos e excluídos aqueles impossibilitados de responder aos questionários devido a algum grau de demência e rebaixamento do nível de consciência; com impossibilidade de realizar as medidas antropométricas; edemaciados e/ou com ascite e gestantes.

Foi utilizado um formulário padronizado para a coleta de dados, contendo dados sociodemográficos, clínicos, bioquímicos, antropométricos e de estilo de vida. As entrevistas e a avaliação nutricional foram realizadas de acordo com a disponibilidade do indivíduo e conduzidas por um nutricionista. Em nenhum momento foi realizado intervenção do pesquisador ou expressão de opinião pessoal que pudesse interferir na resposta ou no resultado da pesquisa.

O diagnóstico da SLHIV foi determinado pela associação de dois parâmetros, um de emagrecimento periférico, avaliado pela escala de gravidade de lipodistrofia e um de acúmulo de gordura central, mensurado pela RCQ. Os pacientes foram então identificados como portado- 
res da SLHIV, se apresentassem alterações nesses dois parâmetros.

Foi aplicada uma escala de gravidade de lipodistrofia adaptada de Carr et al. ${ }^{23}$ para avaliação da presença ou ausência de emagrecimento em regiões periféricas (braços e pernas) onde os pacientes, conforme questionário, classificaram o grau desse emagrecimento, como nenhum (escore 0 ), leve (escore 1), moderada (escore 2) ou grave (escore 3 ). Foi considerado como emagrecimento periférico presente aqueles pacientes que referiram a pontuação de 1-3 nas duas regiões. Enquanto que com o objetivo de identificar a ocorrência de acúmulo de gordura central, foi utilizada a RCQ classificando-a de acordo com o Ministério da Saúde ${ }^{17}$, que adota como ponto de corte os valores superior ou igual a 1,0 para indivíduos do sexo masculino e superior ou igual a 0,85 para o sexo feminino.

Quanto às variáveis sociodemográficas, foram classificados: o sexo em feminino ou masculino, a idade em: 20 a 44 anos e $\geq 45$ anos, o nível de escolaridade em anos de instrução $\mathfrak{E} 9$ anos e > 9 anos, a renda em abaixo de $\mathrm{R} \$ 1.000,00$ e igual ou superior a $\mathrm{R} \$ 1.000,00$ e a etnia em branco e não branco

Para a definição do estado nutricional da amostra utilizou-se o índice de massa corporal (IMC). As medidas de peso e altura foram realizadas segundo a técnica original recomendada por Lohman et al. ${ }^{24}$. E posterior classificação do IMC segundo a Organização Mundial da Saúde ${ }^{25}$, considerando excesso de peso aqueles com IMC $\geq 25 \mathrm{~kg} / \mathrm{m}^{2}$.

Foram considerados contagem de LT-CD4+, carga viral, perfil lipídico, glicemia de jejum, tempo de diagnóstico, classe de TARV e duração de tratamento com a TARV na avaliação das variáveis clínicas e laboratoriais. O LT-CD4+ foi categorizado em $>350$ ou $\mathfrak{E} 350$ células $/ \mathrm{mm}^{3}$, a carga viral classificada em $>40$ ou $\mathfrak{E} 40$ cópias $/ \mathrm{ml}$, o perfil lipídico de acordo com V Diretriz Brasileira de Dislipidemias e Prevenção da Aterosclero$\mathrm{se}^{26}$, a glicemia de jejum foi considerada alterada quando $\geq 100 \mathrm{mg} / \mathrm{dL}$ e sem alteração quando < $100 \mathrm{mg} / \mathrm{dL}$. Enquanto que o tempo de diagnóstico e o tempo de tratamento com TARV foram estratificados em meses (> 60 meses e $\mathfrak{E} 60$ meses), a classe de TARV foi agrupada segundo as classes utilizadas nos esquemas terapêuticos: dois tipos de inibidores de transcripitase reversa análogos nucleosídeos (INTR) associado a um inibidor de transcripitase reversa não nucleosídeo (INNTR) ou dois tipos de ITRNs associado a um inibidor de protease (IP).
Quanto às variáveis de estilo de vida, foram avaliadas ausência ou presença de etilismo independente da quantidade e qualidade de bebida, o tabagismo classificado em fumante, ex-fumante ou que nunca fumou e prática de atividade física, sendo esta segundo International Physical Activity Questionaire (IPAQ) versão curta, que classifica o indivíduo em: Sedentário, pouco ativo, ativo ou muito ativo ${ }^{27}$.

O protocolo deste estudo foi pautado pelas normas éticas para pesquisa envolvendo seres humanos, constantes na resolução 466/12 do Conselho Nacional de Saúde, e foi submetido à avaliação do Comitê de Ética e Pesquisa em Seres Humanos da Universidade Federal de Pernambuco (UFPE), sendo aprovado.

A análise estatística foi realizada através dos programas SPSS (Statistical Package for Social Sciences), versão 13.0 e Stata, versão 7.0. Para avaliar os fatores associados à SLHIV, empregou-se o modelo de regressão de Poisson. Foram calculadas as razões de prevalência pelo método robusto e seu respectivo intervalo de confiança de $95 \%$. O nível de significância adotado foi de $5 \%$. Todas as variáveis que apresentaram $\mathrm{p}<0.20$ na análise bivariada não ajustada, realizada com a utilização do Qui-quadrado com correção de Yates ou do Qui-quadrado de tendência linear foram selecionadas para análise multivariada, utilizando o método stepwise. Ficaram no modelo final unicamente aquelas variáveis com valor de $\mathrm{p}<0.05$.

\section{Resultados}

Foram avaliados 104 PVHIV dos quais 68,3\% eram homens com média de idade de 41,11 ( \pm $11,46)$ anos. Dentre os pacientes avaliados 27,9\% $(\mathrm{n}=29)$ apresentaram a SLHIV (Tabela 1$)$. Houve perdas nas seguintes variáveis: renda, escolaridade, TARV, Carga viral, LT-CD4+, Colesterol total (CT), Triglicerídeo (TG), Lipoproteína de alta densidade (HDL), Lipoproteína de baixa densidade (LDL), excesso de peso e glicemia de jejum.

De acordo com a análise bivariada, observase que dentre as variáveis sociodemográficas e do estilo de vida, apenas o sexo feminino apresentou maior probabilidade de apresentar a SLHIV (RP $=2,31 \mathrm{p}=0,013$ ) (Tabela 1). Quanto às variáveis clínicas, os pacientes com contagem de CD4 $\mathfrak{E} 350(\mathrm{RP}=0,26 \mathrm{p}=0,037)$ apresentaram menor probabilidade de ter a SLHIV. Por outro lado, os pacientes com excesso de peso apresentaram três vezes mais chance de ter SLHIV $(\mathrm{RP}=3,20 \mathrm{p}=$ 
Tabela 1. Associação da síndrome lipodistrófica do HIV (SLHIV) com variáveis sociodemográficas e do estilo de vida de pacientes HIV+ atendidos em ambulatório de um hospital universitário do estado de Pernambuco. Recife/PE, Brasil,2017.

\begin{tabular}{|c|c|c|c|c|c|c|c|c|c|}
\hline \multirow{3}{*}{$\begin{array}{l}\text { Variáveis } \\
\text { Sexo }\end{array}$} & \multicolumn{9}{|c|}{ Síndrome Lipodistrofica do HIV } \\
\hline & \multicolumn{2}{|c|}{ Sim } & \multicolumn{2}{|c|}{ Não } & \multicolumn{2}{|c|}{ Total } & \multirow[t]{2}{*}{$\dagger \mathbf{R P}$} & \multirow{2}{*}{$\mathrm{IC}_{95 \%}$} & \multirow[t]{2}{*}{$\mathbf{P}$} \\
\hline & $\mathbf{N}$ & $\%$ & $\mathbf{N}$ & $\%$ & $\mathbf{N}$ & $\%$ & & & \\
\hline Feminino & 15 & 45,5 & 18 & 54,5 & 33 & 31,7 & 2,31 & $1,27-9,23$ & $0,013^{\mathrm{a}}$ \\
\hline Masculino & 14 & 19,7 & 57 & 80,3 & 71 & 68,3 & 1,00 & & \\
\hline \multicolumn{10}{|l|}{ Idade (anos) } \\
\hline$\geq 45$ & 15 & 37,5 & 25 & 62,5 & 40 & 38,5 & 1,71 & $0,93-3,16$ & $0,133^{\mathrm{a}}$ \\
\hline $20-44$ & 14 & 21,9 & 50 & 78,1 & 64 & 61,5 & 1,00 & & \\
\hline \multicolumn{10}{|l|}{ Renda familiar (reais) } \\
\hline$\leq 1000,00$ & 16 & 30,8 & 36 & 69,2 & 52 & 51,0 & 1,59 & $0,85-2,97$ & $0,216^{\mathrm{a}}$ \\
\hline$>1000,00$ & 12 & 24,0 & 38 & 76,0 & 50 & 49,0 & 1,00 & & \\
\hline \multicolumn{10}{|l|}{ Escolaridade (anos) } \\
\hline$\leq 9$ & 13 & 28,3 & 33 & 71,7 & 46 & 46 & 1,09 & $0,57-2,08$ & $0,971^{\mathrm{a}}$ \\
\hline$>10$ & 14 & 25,9 & 40 & 74,1 & 54 & 54 & 1,00 & & \\
\hline \multicolumn{10}{|l|}{ Ingestão Alcool** } \\
\hline Sim & 11 & 22,4 & 38 & 77,6 & 49 & 47,1 & 0,69 & $0,36-1,31$ & $0,343^{\mathrm{a}}$ \\
\hline Não & 18 & 32,7 & 37 & 67,3 & 55 & 52,9 & 1,00 & & \\
\hline \multicolumn{10}{|l|}{ Tabagismo } \\
\hline Fumante & 04 & 20,0 & 16 & 80,0 & 20 & 19,2 & 0,74 & $0,23-1,95$ & $0,491^{\mathrm{b}}$ \\
\hline Ex fumante & 08 & 38,1 & 13 & 61,9 & 21 & 20,2 & 1,41 & $0,72-2,79$ & \\
\hline Nunca fumou & 17 & 27,0 & 46 & 73,0 & 63 & 60,6 & 1,00 & & \\
\hline \multicolumn{10}{|l|}{ Nível de Atividade Física } \\
\hline Sedentário/Pouco ativo & 08 & 25,0 & 24 & 75,0 & 32 & 30,8 & 0,94 & $0,33-2,63$ & $0,967^{\mathrm{b}}$ \\
\hline Ativo & 17 & 29.8 & 40 & 70,2 & 57 & 54,8 & 1,12 & $0,44-2,83$ & \\
\hline Muito ativo & 04 & 26,7 & 11 & 73,3 & 15 & 14,4 & 1,00 & & \\
\hline
\end{tabular}

0,002) (Tabela 2). Outras variáveis, tais como: idade $^{3} 45$ anos, presença de hipercolesterolemia, tempo de diagnóstico e tempo de TARV $>60$ meses entraram no modelo por apresentarem $\mathrm{p}<$ 0.20 na análise bivariada não ajustada.

Quanto aos resultados da regressão de Poisson e os efeitos ajustados das variáveis explanatórias, em relação à SLHIV, observa-se que o sexo feminino $\left(\mathrm{RP}_{\text {ajustada }}=2,16 \mathrm{IC}_{95 \%} 1,43-3,39\right)$, o excesso de peso $\left(\mathrm{RP}_{\text {ajustada }}=2,23 \mathrm{IC}_{95 \%} 1,35-2,65\right)$ e o maior tempo de uso dos antirretrovirais $\left(\mathrm{RP}_{\text {ajustad }}\right.$ $\left.=1,64 \mathrm{IC}_{95 \%} 1,16-2,78\right)$ permaneceram positivamente associados à síndrome. Por outro lado, foi observada uma associação negativa com a contagem de CD4 $2350\left(\mathrm{RP}_{\text {ajustada }}=0,39 \mathrm{IC}_{95 \%} 0,10\right.$ 0,97) (Tabela 3).

\section{Discussão}

As principais pesquisas sobre as complicações metabólicas do uso da TARV foram conduzidas principalmente em países de alta renda. De forma crescente, tais pesquisas em países de média e baixa renda vêm sendo evidenciadas, avaliando os efeitos do uso dos antirretrovirais a curto e a longo prazos ${ }^{28}$.

Conhecer as condições de saúde, em particular a presença de complicações, dos portadores de HIV na população a qual se presta assistência, permite a identificação dos pacientes em risco e direciona a implantação de medidas preventivas. Várias dessas complicações, em particular a lipodistrofia, podem predispor os indivíduos ao risco cardiovascular, impactando na qualidade de vida e na adesão ao tratamento ${ }^{29}$.

Neste contexto, nos propomos avaliar a presença de SLHIV naqueles pacientes em uso de TARV. A frequência de SLHIV de 27,9\% aqui encontrada foi semelhante aos resultados de Della Justina $^{30}$, em um estudo transversal brasileiro, utilizando também método diagnóstico subjetivo associado a medidas objetivas em pacientes ambulatoriais em uso de TARV, que encontrou 
Tabela 2. Associação da síndrome lipodistrófica do HIV (SLHIV) com variáveis clínicas e antropométricas de pacientes HIV+ atendidos em ambulatório de um hospital universitário do estado de Pernambuco. Recife/PE, Brasil,2017.

\begin{tabular}{|c|c|c|c|c|c|c|c|c|c|}
\hline \multirow{3}{*}{$\begin{array}{c}\text { Variáveis } \\
\text { Tempo diagnóstico (meses) }\end{array}$} & \multicolumn{9}{|c|}{$\begin{array}{c}\text { Síndrome Lipodistrofica } \\
\text { do HIV }\end{array}$} \\
\hline & \multicolumn{2}{|c|}{ Sim } & \multicolumn{2}{|c|}{ Não } & \multicolumn{2}{|c|}{ Total } & \multirow[t]{2}{*}{$\dagger \mathbf{R P}$} & \multirow[t]{2}{*}{ IC $_{95 \%}$} & \multirow[t]{2}{*}{$\mathbf{p}^{\mathbf{a}}$} \\
\hline & $\mathbf{N}$ & $\%$ & $\mathbf{N}$ & $\%$ & $\mathbf{N}$ & $\%$ & & & \\
\hline$>60$ & 17 & 37 & 29 & 63 & 46 & 44,2 & 1,79 & $0,95-3,35$ & 0,106 \\
\hline$\leq 60$ & 12 & 20,7 & 46 & 79,3 & 58 & 55,8 & 1,00 & & \\
\hline \multicolumn{10}{|l|}{ Tempo uso de TARV (meses) } \\
\hline$>60$ & 16 & 38,1 & 26 & 61,9 & 42 & 40,4 & 1,82 & $0,98-3,37$ & 0,091 \\
\hline$\leq 60$ & 13 & 21 & 49 & 79,0 & 62 & 59,6 & 1,00 & & \\
\hline \multicolumn{10}{|l|}{ Tipo de TARV } \\
\hline 2 INTR + 1 INNTR & 17 & 30,4 & 39 & 69,6 & 56 & 59,6 & 1,15 & $0,59-2,24$ & 0,847 \\
\hline 2 INTR + 1 IP & 10 & 26,3 & 28 & 73,7 & 38 & 40,4 & 1,00 & & \\
\hline \multicolumn{10}{|l|}{ Carga viral (cópias/ml) } \\
\hline$>40$ & 04 & 28,6 & 10 & 71,4 & 14 & 16,3 & 0,98 & $0,40-2,42$ & 0,782 \\
\hline$\leq 40$ & 21 & 29,2 & 51 & 70,8 & 72 & 83,7 & 1,00 & & \\
\hline \multicolumn{10}{|l|}{ Contagem CD4 (céls/mm³) } \\
\hline$\leq 350$ & 02 & 9,1 & 20 & 90,9 & 22 & 25,3 & 0,26 & $0,07-1,00$ & 0,037 \\
\hline$>350$ & 23 & 35,4 & 42 & 64,6 & 65 & 74,7 & 1,00 & & \\
\hline \multicolumn{10}{|l|}{ Hipercolesterolemia (mg/dL) } \\
\hline $\operatorname{Sim}$ & 12 & 46,2 & 14 & 53,8 & 26 & 31,7 & 1,99 & $1,06-3,74$ & 0,065 \\
\hline Não & 13 & 23,2 & 43 & 76,8 & 56 & 68,3 & 1,00 & & \\
\hline \multicolumn{10}{|l|}{ Hipertrigliceridemia $(\mathrm{mg} / \mathrm{dL})$} \\
\hline $\operatorname{Sim}$ & 09 & 25,0 & 27 & 75,0 & 36 & 42,9 & 0,80 & $0,40-1,62$ & 0,701 \\
\hline Não & 15 & 31,3 & 33 & 68,8 & 48 & 57,1 & 1,00 & & \\
\hline \multicolumn{10}{|l|}{ HDL baixo (mg/dL) } \\
\hline Sim & 18 & 27,7 & 47 & 72,3 & 65 & 83,3 & 0,72 & $0,33-1,59$ & 0,320 \\
\hline Não & 05 & 38,5 & 08 & 61,5 & 13 & 16,6 & 1,00 & & \\
\hline \multicolumn{10}{|l|}{ LDL elevado (mg/dL) } \\
\hline Sim & 14 & 33.3 & 28 & 66,7 & 42 & 53,2 & 1,50 & $0,71-3,16$ & 0,404 \\
\hline Não & 08 & 22,2 & 28 & 77,8 & 36 & 46,8 & 1,00 & & \\
\hline \multicolumn{10}{|l|}{ Excesso de Peso $\left(\mathrm{kg} / \mathrm{m}^{2}\right)$} \\
\hline Sim & 22 & 43,1 & 29 & 56,9 & 51 & 49,5 & 3,20 & $1,50-6,84$ & 0,002 \\
\hline Não & 07 & 13,5 & 45 & 86,5 & 52 & 50,5 & 1,00 & & \\
\hline \multicolumn{10}{|c|}{ Alteração na Glicemia de Jejum(mg/dL) } \\
\hline Sim & 18 & 28,1 & 46 & 71,9 & 64 & 77,1 & 0,76 & $0,38-1,55$ & 0,658 \\
\hline Não & 7 & 36,8 & 12 & 63,2 & 19 & 22,9 & 1,00 & & \\
\hline
\end{tabular}

valores de $32,4 \%$. Estudos brasileiros obtiveram prevalências variando entre 32 e 68\%, semelhante a estudos realizados em outros países com variação entre 30 e $62 \%$. Esse grande diferencial com relação à prevalência de SLHIV pode ser explicada pela ausência na literatura de consenso quanto ao diagnóstico da síndrome $e^{4,12,31,32}$.

Foi observado que as mulheres apresentaram uma probabilidade 2,16 vezes maior de apresentar a SLHIV. Outros autores, avaliando essa associação, observaram resultados semelhan- tes $\mathrm{s}^{11,33-35}$ com uma probabilidade 2,5 vezes maior em mulheres ${ }^{34-37}$, fato que pode ser atribuível a diferenças fisiológicas relacionadas ao gênero. Há evidências sobre estas diferenças que apontam associação do polimorfismo do gene do receptor de estrógeno nas células adiposas de mulheres, sugerindo uma predisposição genética para o desenvolvimento de lipoatrofia e/ou acúmulo de gordura corporal ${ }^{21,38,39}$. Sabendo disso, é importante voltar à atenção a esse grupo, porque, principalmente em países subdesenvolvidos, as 
Tabela 3. Regressão de Poisson com razão de prevalência bruta e ajustada (RP) dos fatores associados a síndrome lipodistrófica do HIV de pacientes HIV+ atendidos em ambulatório de um hospital universitário do estado de Pernambuco. Recife/PE, Brasil,2017.

\begin{tabular}{lcccccc}
\hline \multicolumn{1}{c}{ Variáveis } & \multicolumn{6}{c}{ Síndrome Lipodistrófica do HIV } \\
\cline { 2 - 7 } & $\begin{array}{c}\text { RP } \dagger \\
\text { bruta }\end{array}$ & IC $_{95 \%}$ & $\mathbf{p}$ & $\begin{array}{c}\text { RP } \dagger \\
\text { ajustada }\end{array}$ & IC $_{95 \%}$ & $\mathbf{p}$ \\
\hline Sexo feminino & 2,31 & $1,27-9,23$ & 0,013 & 2,16 & $1,43-3,39$ & 0,022 \\
Idade $\geq 45$ anos & 1,71 & $0,93-3,16$ & 0,133 & 1,14 & $0,88-3,04$ & 0,413 \\
Tempo de diagnostico $>$ 60 meses & 1,79 & $0,95-3,35$ & 0,106 & 1,84 & $0,56-3,22$ & 0,214 \\
Tempo de TARV $>$ 60 meses & 1,82 & $0,98-3,37$ & 0,091 & 1,64 & $1,16-2,78$ & 0,042 \\
Contagem de CD4 $\leq 350$ & 0,26 & $0,07-1,00$ & 0,037 & 0,39 & $0,10-0,97$ & 0,034 \\
Excesso de peso & 3,20 & $1,50-6,84$ & 0,002 & 2,23 & $1,35-2,65$ & 0,000 \\
Hipercolesterolemia & 1,99 & $1,06-3,74$ & 0,065 & 1,43 & $0,53-3,24$ & 0,112 \\
\hline Regressão de Poisson $\dagger \mathrm{RP}=$ Razão de Prevalência IC ${ }_{95 \%}=$ Intervalo de Confiança de 95\%; TARV: Terapia antirretroviral.
\end{tabular}

mulheres podem ter menos probabilidade de receber cuidados de saúde, educação e apoio durante o tratamento, impactando ainda mais nas consequências metabólicas ${ }^{40,41}$.

Quando avaliamos o nível de atividade física, este não apresentou associação com a SLHIV. Todavia, dados na literatura constatam que há uma alta incidência de sedentarismo em indivíduos diagnosticados com SLHIV ${ }^{30,34,42}$, indicando que a prática de atividade física poderia ser um fator protetor em relação à sua ocorrência ${ }^{43}$. Sabe-se que a prática de exercício físico associado à dieta pode reduzir a progressão da síndrome e diminuir seu impacto na saúde e na qualidade de vida. Foi demonstrado que indivíduos infectados pelo HIV e com lipodistrofia, após um programa de exercícios físicos durante seis meses, apresentaram melhora cardiorrespiratória, metabólica e na qualidade de vida, comparado aos não exer$\operatorname{citados}^{44,45}$.

Quanto aos parâmetros clínicos da infecção pelo HIV, os pacientes com LT-CD4 $+\leq 350$ céls $/ \mathrm{mm}^{3}$ tinham menor chance de desenvolver SLHIV, mesmo após ajuste. Em concordância com esses achados, Lichtenstein et al. ${ }^{46}$, em uma coorte com 1077 pacientes em idade média de 41 anos (85\% do sexo masculino), e Segatto et al. ${ }^{42}$ avaliando 42 pacientes ( $55 \%$ do sexo masculino), verificaram associação entre a presença da síndrome com maiores níveis de L-TCD4+ em indivíduos em uso de TARV. Justificando que o uso dessa terapia tende a melhorar a condição imunológica, com uma consequente progressão mais lenta da doença e menor incidência de morte, contudo está associado ao aparecimento da SLHIV $34,42,47-49$.

Entretanto Silva ${ }^{50}$, avaliando 219 pacientes que não estavam em uso regular de antirretro- virais, encontrou associação entre menor $\mathrm{CD} 4 \mathrm{e}$ maior IMC, assim como de maior percentual de gordura corporal. Esses achados podem ser resultado dos efeitos deletérios oriundos da própria infecção pelo HIV provavelmente como consequencia da ativação de macrófagos nas células adiposas, o que poderia aumentar a inflamação local e sistêmica ${ }^{30,51}$.

Também foi possível identificar que o maior tempo de uso de TARV associou-se com o desenvolvimento da SLHIV. Da mesma forma Soares e Costa ${ }^{52}$, em uma coorte com 219 pacientes, encontraram que homens e mulheres com SLHIV apresentaram maior tempo de uso de TARV (de 4 a 7 anos). Visto que no Brasil há uma importante atuação na disponibilização desses medicamentos e estes colaboram para a melhora da condição imune, devemos então levar em consideração que as PVHIV em uso de TARV podem estar sujeitos ao aumento na sobrevida e além dos efeitos colaterais provocados pela TARV (redistribuição de gordura corporal e anormalidades metabólicas), exposição à fatores fisiológicos inerentes ao próprio envelhecimento e fatores externos, como um estilo de vida sedentário e de alto consumo energético que contribuem para o aumento do risco cardiovascular ${ }^{2,17,34,53-55}$.

Quanto ao tipo de TARV, não foi encontrada associação estatisticamente significante. Diferente dos nossos achados, a literatura descreve que a SLHIV está entre os mais prevalentes e preocupantes efeitos colaterais de alguns tipos de TARV ${ }^{56}$. Inicialmente atribuía-se o desenvolvimento da síndrome aos IP's ${ }^{46,57}$, porém com a introdução de outras classes de antirretroviais, observou-se que os ITRN, especialmente a estavudina e a zidovudina, também estariam associados com a síndrome $e^{17,58-60}$. 
$\mathrm{O}$ excesso de peso tem se mostrado presente em $35-45 \%$ da população com SLHIV 22,61,62, corroborando com nossos achados de $43 \%$ (p < 0,002 ). Arruda Junior et al..$^{63}$, em um estudo realizado na cidade do Recife, analisando 958 pacientes (61\% do gênero masculino), verificaram que a presença de SLHIV estava associada com sobrepeso/obesidade $(52,7 \%)$ e que estes tinham maior tempo de uso de TARV ( $>24$ meses). Vale salientar que o estado nutricional de sobrepeso/ obesidade permaneceu associado à ocorrência da síndrome mesmo após eliminação dos fatores de confusão. Mariz et al. ${ }^{64}$ relatam que esse aumento da prevalência de obesidade em PVHIV está associado à redução significativa de doenças oportunistas e cronicidade do HIV, como consequência do uso da TARV que atua diretamente contra etapas importantes da replicação do HIV ${ }^{65}$. Além disso, outros estudos também demonstraram a presença de excesso de peso nesta população como um dado preocupante, uma vez que o uso de TARV associado com excesso de peso e acúmulo de gordura predispõe esses pacientes ao desenvolvimento da síndrome metabólica e de doenças cardiovasculares ${ }^{66}$.

Não encontramos associação entre a presença da SLHIV e a presença de dislipidemia e alteração na glicemia de jejum, no entanto vários autores destacam essa relação na literatura ${ }^{67-69}$. Em um estudo transversal na Tailândia, com 278 pacientes infectados pelo HIV, $93 \%$ dos pacientes com SLHIV tinha pelo menos uma anormalidade metabólica (dislipidemia, intolerância a glicose ou resistência à insulina $)^{70}$, enquanto que outras grandes bases de dados não mostraram essa associação quando os resultados foram ajustados para fatores de risco tradicionais ${ }^{67,68,71,72}$.

Como limitações do estudo podemos destacar o pequeno número amostral, a falta de consenso diagnóstico, a ausência de dados referentes à adesão a TARV, a coleta de dados que foi realizada em único centro de referência, que apresenta características próprias e inerentes à realidade local, limitando assim, a validade externa absoluta dos seus achados. No entanto, os resultados deste estudo nos possibilitam conhecer a realidade de saúde dos pacientes neste serviço e prestar a essa população melhor assistência e acompanhamento, além de serem usados na prática clínica para comparação e avaliação dos PVHIV.

A partir desses resultados, podemos destacar que a frequência da síndrome foi semelhante aos valores descritos na literatura, reforçando que essa distribuição corporal anômala pode ser identificada nas PVHIV em uso de TARV. Além disso, destaca a associação da síndrome com os fatores de risco considerados modificáveis, como obesidade/excesso de peso, representando para esse grupo maior risco de desenvolvimento de outras comorbidades como o DM e a hipertensão.

Este trabalho possibilitou diagnosticar a SLHIV, assim como também identificar os principais grupos associados à síndrome através de métodos simples e aplicáveis na prática clínica. A partir disso, é possível traçar medidas de prevenção e intervenção precoce de acordo com a realidade encontrada. Tais medidas contribuem possibilitando a melhora das condições de saúde e concomitante aumento da expectativa de vida com qualidade desses indivíduos.

\section{Colaboradores}

LLG Silva trabalhou no desenvolvimento da escrita do trabalho; EM Santos, coleta de dados; LCP Nascimento, tabulação de dados; M Cavalcanti, Correção da introdução e desenvolvimento; MCL Luz, coorientação do trabalho e correções finais; PC Cabral, orientação e estatística. IKG Arruda, revisão da análise estatística. 


\section{Referências}

1. Weiss R. Special Anniversary Review: Twenty-five years of human immunodeficiency virus research: successes and challenges. Clin Exp Immunol 2008; 152(2):201-210.

2. Brasil. Ministério da Saúde (MS). Boletim Epidemiológico - Aids e DST 2015; 1(1):1-100.

3. Carr A, Samaras K, Burton S, Law M, Freund J, Chisholm DJE, Cooper DA. A syndrome of peripheral lipodystrophy, hyperlipidaemia and insulin resistance in patients receiving HIV protease inhibitors. AIDS 1998; 12(7):F51-F58.

4. Santos CP, Felipe YX, Braga PE, Ramos D, Lima ROE, Segurado AC. Self-perception of body changes in persons living with HIV/AIDS: prevalence and associated factors. AIDS 2005; 19(Supl. 4):S14-S21.

5. Tien PC, Cole SR, Williams CM, Li, R, Justman JE, Cohen MH, Young M, Rubin N, Augenbraun ME, Grunfeld C. Incidence of Lipoatrophy and Lipohypertrophy in the Women's Interagency HIV Study. J Acquir Immune Defic Syndr 2003; 34(5):461-466.

6. Wohl DA, Mccomsey G, Tebas P, Brown TT, Glesby MJ, Reeds D, Shikuma C, Mulligan K, Dube M, Wininger D, Huang J, Revuelta M, Currier J, Swindells S, Fichtenbaum C, Basar M, Tungsiripat M, Meyer W, Weihe JE, Wanke C. Current Concepts in the Diagnosis and Management of Metabolic Complications of HIV Infection and Its Therapy. Clin Infect Dis 2006; 43(5):645-653.

7. Jacobson DL, Knox T, Spiegelman D, Skinner S, Gorbach S, Wanke C. Prevalence of, Evolution of, and Risk Factors for Fat Atrophy and Fat Deposition in a Cohort of HIV-Infected Men and Women. Clin Infect Dis 2005; 40(12):1837-1845.

8. Carr A. Lactic Acidemia in Infection with Human Immunodeficiency Virus. Clin Infect Dis 2003; 36(s2):S96-S100.

9. Heath KV, Hogg RS, Chan KJ, Harris M, Montessori V, O’shaughnessy MV, Montaner JSG. Lipodystrophy -associated morphological, cholesterol and triglyceride abnormalities in a population-based HIV/AIDS treatment database. AIDS 2001; 15(2):231-239.

10. Leitz G, Robinson P. The development of lipodystrophy on a protease inhibitor-sparing highly active antiretroviral therapy regimen. AIDS 2000; 14(4):468.

11. Mutimura E, Stewart A, Crowther NJ. Assessment of quality of life in HAART-treated HIV-positive subjects with body fat redistribution in Rwanda. AIDS Res Ther 2007; 4(1):19.

12. Monnerat BZ, Cerutti Junior C, Caniçali SC, Motta TR. Clinical and biochemical evaluation of HIV-related lipodystrophy in an ambulatory population from the Hospital Universitário Cassiano Antonio de Morais, Vitória, ES, Brazil. Braz J Infect Dis 2008; 12(4):364-368.

13. Anjos EMD, Pfrimer K, Machado AA, Cunha SFDC, Salomão RG, Monteiro JP. Nutritional and metabolic status of HIV-positive patients with lipodystrophy during one year of follow-up. Clinics 2011; 66(3):407410.

14. Kroll AF, Sprinz E, Leal SC, Labrêa MDG, Setúbal S. Prevalence of obesity and cardiovascular risk in patients with HIV/AIDS in Porto Alegre, Brazil. Arq Bras Endocrinol Metabol 2012; 56(2):137-141.
15. Joly V, Flandre P, Meiffredy V, Leturque N, Harel M, Aboulker J, Yeni P. Increased risk of lipoatrophy under stavudine in HIV-1-infected patients. AIDS 2002; 16(18):2447-2454.

16. Milinkovic A, Martinez E. Current perspectives on HIV-associated lipodystrophy syndrome. J Antimicrob Chemother 2005; 56(1):6-9.

17. Brasil. Ministério da Saúde (MS). Sindrome Lipodistrófica em HIV. Brasília: MS; 2011.

18. Florindo AA, Latorre MRDO, Santos ECM, Borelli A, Rocha MS, Segurado AAC. Validation of methods for estimating HIV/AIDS patients' body fat. Rev Saude Publica 2004; 38(5):643-649.

19. Hengel R, Watts N, Lennox J. Benign symmetric lipomatosis associated with protease inhibitors. Lancet 1997; 350(9091):1596.

20. Carvalho EH, Miranda Filho DDB, Ximenes RADA, Albuquerque MDFPM, Melo HRL, Gelenske T, Medeiros ZDB, Montarroyos EU, Bandeira F. Prevalence of Hyperapolipoprotein B and Associations with Other Cardiovascular Risk Factors Among Human Immunodeficiency Virus-Infected Patients in Pernambuco, Brazil. Met Synd Rel Dis 2010; 8(5):403-410.

21. Gasparotto AS, Sprinz E, Lazzaretti RK, Kuhmmer R, Silveira JM, Basso RP, Pinheiro CA, Silveira MF, Ribeiro JP, Mattevi VS. Genetic polymorphisms in estrogen receptors and sexual dimorphism in fat redistribution in HIV-infected patients on HAART. AIDS 2012; 26(1):19-26.

22. Soares LR, Silva DCD, Gonsalez CR, Batista FG, Fonseca LAM, Duarte AJE, Casseb J. Discordance between body mass index and anthropometric measurements among HIV-1-infected patients on antiretroviral therapy and with lipoatrophy/lipohypertrophy syndrome. Rev Inst Med Trop São Paulo 2015; 57(2):105-110.

23. Carr A, Samaras K, Burton S, Law M, Freund J, Chisholm D, Cooper DA. A syndrome of peripheral lipodystrophy, hyperlipidaemia and insulin resistance in patients receiving HIV protease inhibitors. AIDS 1998; 12(7):F51-F58.

24. Lohman TG, Martorell R, Roche AF. Anthropometric standardization reference manual. Human Kinetics Books. Champaign: Ill; 1988.

25. World Health Organization (WHO). Obesity. Preventing and Managing the Global Epidemic. Report of a WHO Consultation on Obesity. Geneva: WHO; 1997.

26. Xavier HT, Izar MC, Faria Neto JR, Assad MH, Rocha VZ, Sposito AC, Fonseca FA, dos Santos JE, Santos RD, Bertolami MC, Faludi AA, Martinez TLR, Diament J, Guimarães A, Forti NA, Moriguchi E, Chagas ACP, Coelho OR, Ramires JAF. V Diretriz Brasileira de Dislipidemias e Prevenção da Aterosclerose. Arq Bras Cardiol 2013; 101(4 Supl. 1):1-20.

27. Finkelstein JL, Gala P, Rochford R, Glesby MJ, Mehta S. HIV/AIDS and lipodystrophy: Implications for clinical management in resource-limited settings. $J$ Int AIDS Soc 2015; 18(1):19033.

28. Grinspoon S, Carr A. Cardiovascular Risk and BodyFat Abnormalities in HIV-Infected Adults. $N$ Engl J Med 2005; 352(1):48-62.

29. International Physical Activity Questionnaire (IPAQ) [Internet]. 2001 [cited 2016 Oct 26]. Available from: http://www.ipaq.ki.se/ipaq.htm 
30. Della Justina LB. Prevalence of HIV-Associated Lipodystrophy in Brazil: A Systematic Review of the Literature. J AIDS Clin 2014; 5:9.

31. Ceccato M, Bonolo P, Souza Neto A, Araújo F, Freitas M. Antiretroviral therapy-associated dyslipidemia in patients from a reference center in Brazil. Braz J Med Biol Res 2011; 44(11):1177-1183.

32. Tien P, Grunfeld C. What is HIV-associated lipodystrophy? Defining fat distribution changes in HIV infection. Curr Opin Infect Dis 2004; 17(1):27-32.

33. Dutra CDT, Moura AKM, Saraiva DA, Dias RM, Oliveira SHD, Frazão ADGF, Libonati RMF. Fatores de risco para doenças cardiovasculares em pacientes com síndrome lipodistrófica do HIV. GઐS 2012; 3(3):558.

34. Sacilotto LB. Composição corporal e componentes da síndrome metabólica nos diferentes subtipos de lipodistrofia associada ao HIV [dissertação]. Botucatu: Universidade Estadual Paulista; 2017

35. Menezes CN, Maskew M, Sanne I, Crowther NJ, Raal FJ. A longitudinal study of stavudine-associated toxicities in a large cohort of South African HIV infected subjects. BMC Infect Dis 2011; 11:244

36. van Griensven J, Zachariah R, Mugabo J, Reid T. Weight loss after the first year of stavudine-containing antiretroviral therapy and its association with lipoatrophy, virological failure, adherence and CD4 counts at primary health care level in Kigali, Rwanda. Trans $R$ Soc Trop Med Hyg 2010; 104(12):751-757.

37. Silva ICD, Sampaio E, Almeida M, Ney Freire, A, Ramos L, Medeiros JMB. Perfil metabólico, antropométrico e lipodistrofia em pessoas vivendo com hiv/aids em uso de terapia antirretroviral. Nutr Clín Diet Hosp 2016; 36(3):38-44.

38. Pedersen SB, Bruun JM, Hube F, Kristensen K, Hauner $\mathrm{H}$, Richelsen B. Demonstration of estrogen receptor subtypes and in human adipose tissue: influences of adipose cell differentiation and fat depot localization. Mol Cell Endocrinol 2001; 182(1):27-37.

39. Nyblade L, Pande R, Mathur S, MacQuarrie K, Kidd R, Banteyerga H, Kidanu A, Kilonzo G, Mbwambo J, Bond V. Disentangling HIV and stigma in Ethiopia, Tanzania and Zambia. Washington: International Center for Research on Women; 2003.

40. Mutimura E, Stewart A, Crowther NJ. Assessment of quality of life in HAART-treated HIV-positive subjects with body fat redistribution in Rwanda. AIDS Res Ther 2007; 4(1):19.

41. Finkelstein JL, Gala P, Rochford R, Glesby MJ, Mehta S. HIV/AIDS and lipodystrophy: Implications for clinical management in resource-limited settings. J Int Aids Soc 2015; 18(1):19033.

42. Segatto AFM, Freitas Junior IF, Santos VRD, Alves KCP, Barbosa DA, Portelinha Filho AM, Monteiro HL. Lipodystrophy in HIV/AIDS patients with different levels of physical activity while on antiretroviral therapy. Rev Soc Bras Med Tro 2011; 44(4):420-424.

43. Mutimura E, Stewart A, Crowther NJ, Yarasheski KE, Cade WT. The effects of exercise training on quality of life in HAART-treated HIV-positive Rwandan subjects with body fat redistribution. Qual Life Res 2008; 17(3):377-385.
44. Mutimura E, Crowther NJ, Cade TW, Yarasheski KE, Stewart A. Exercise Training Reduces Central Adiposity and Improves Metabolic Indices in HAART-Treated HIV-Positive Subjects in Rwanda: A Randomized Controlled Trial. AIDS Res Hum Retroviruses 2008; 24(1):15-23.

45. Collins E, Wagner C, Walmsley S. Psychosocial impact of the lipodystrophy syndrome in HIV infection. AIDS Read 2000; 10(9):546-550.

46. Lichtenstein KA, Ward DJ, Moorman AC, Delaney KM, Young B, Palella FJ, Rhodes PH, Wood KC Holmberg SD. Clinical assessment of HIV-associated lipodystrophy in an ambulatory population. AIDS 2001; 15(11):1389-1398.

47. Soares FMG, Costa IMC. Treatment of HIV-associated facial lipoatrophy: impact on infection progression assessed by viral load and CD4 count. An Bras Dermatol 2013; 88(4):570-577.

48. Santos MRD, Araújo JVD, Miranda Filho DDB, Ximenes RADA, Santos Júnior BJD. Perfil lípidico dos pacientes HIV positivos em uso da terapia antirretroviral. Rev Bras Multi 2017; 20(1):61.

49. Alencastro PR, Barcellos NT, Wolff FH, Ikeda MLR, Schuelter-Trevisol F, Brandão ABM, Fuchs SC. People living with HIV on ART have accurate perception of lipodystrophy signs: a cross-sectional study. BMC Res Notes 2017; 10:1.

50. Silva LFG. Fatores clínicos e laboratoriais da doença pelo HIV na composição corporal, força muscular, nível de atividade física, lipodistrofia e sua repercussão na qualidade de vida [tese]. Belém: Universidade Federal do Pará; 2014.

51. Crowe S, Westhorpe C, Mukhamedova N, Jaworowski A, Sviridov D, Bukrinsky M. The macrophage: the intersection between HIV infection and atherosclerosis. J Leukoc Biol 2009; 87(4):589-598.

52. Soares FMG, Costa IMC. Lipoatrofia facial associada ao HIV/AIDS: do advento ao sconhecimentos atuais Anais Brasileiros de Dermatologia 2011; 86(5):843864.

53. Oliveira JF, Silva RX, Silva RMB, Manita OMG, Martelli A, Delbim LR. Benefícios do Exercício Físico como Terapia Alternativa para Indivíduos Portadores de HIV/AIDS. Rev Saúde em Foco 2016; 3(2):3-16.

54. Capeau J. From lipodystrophy and insulin resistance to metabolic syndrome: HIV infection, treatment and aging. Curr Opin HIV AIDS 2007; 2(4):247-252.

55. Guimarães MMM, Greco DB, Ribeiro-Olivera Júnior A, Penido MG, Machado LJC. Distribuição da gordura corporal e perfis lipídico e glicêmico de pacientes infectados pelo HIV. Arq Bras Endocrinol Metab 2000; 51(1):42-51.

56. Martínez E, Mocroft A, García-Viejo MA, PérezCuevas JB, Blanco JL, Mallolas J, Bianchi L, Conget I, Blanch J, Phillips A, Gatell JM. Risk of lipodystrophy in HIV-1-infected patients treated with protease inhibitors: a prospective cohort study. Lancet 2001; 357(9256):592-598.

57. Palmer M, Chersich M, Moultrie H, Kuhn L, Fairlie L, Meyers T. Frequency of stavudine substitution due to toxicity in children receiving antiretroviral treatment in sub-Saharan Africa. AIDS 2013; 27(5):781-785. 
58. Pontes CMM. Distúrbios metabólicos associados a infecção pelo HIV/AIDS: prevalência em pacientes ambulatórios seguidos em hospital de referência do estado do Ceará, Brasil [dissertação]. Fortaleza: Universidade Federal do Ceará; 2010.

59. Han SH, Zhou J, Saghayam S, Vanar S, Phanuphak N, Chen YA, Sirisanthana T, Sungkanuparph S, Lee CK, Pujari S, Li PC, Oka S, Saphonn V, Zhang F, Merati TP, Law MG, Choi JY. Prevalence of and risk factors for lipodystrophy among HIV-infected patients receiving combined antiretroviral treatment in the Asia-Pacific region: results from the TREAT Asia HIV Observational Database (TAHOD). Endocr J 2011; 58(6):475484.

60. Mallal SA, John M, Moore CB, James IR, McKinnon EJ. Contribution of nucleoside analogue reverse transcriptase inhibitors to subcutaneous fat wasting in patients with HIV infection. AIDS 2000; 14(10):1309-1316.

61. Santos TMP, Silva DB, Franco TM, Santos VR, Mendonça J, Santos Júnior JA, Novais GB, Costa D. Lipodystrophy and the relationship with cardiovascular risk factors and metabolic syndrome in HIV-infected patients. Nutr Clín Diet Hosp 2017; 37(2):12-20.

62. Oliveira TGB. Lipodistrofia em indivíduos vivendo com HIV/AIDS: fatores de risco para lipoatrofia e acúmulo de gordura corporal e associação com síndrome metabólica. Um estudo de coorte [tese]. Recife: Universidade Federal de Pernambuco; 2014.

63. Arruda Júnior ERD, Lacerda HR, Moura LCRV, Albuquerque MDFPMD, Miranda Filho, DDB, Diniz GTN, Albuquerque VMGD, Amaral JCZ, Monteiro VS, Ximenes RADA. Perfil dos pacientes com hipertensão arterial incluídos em uma coorte com HIV/ AIDS em Pernambuco, Brasil. Arq Bras Cardiol 2010; 95(5):640-647.

64. Mariz CDA, Albuquerque MDFPMD, Ximenes RADA, Melo HRLD, Bandeira F, Oliveira TGBE, Carvalho EHD, Silva APD, Miranda Filho, DDB. Body mass index in individuals with HIV infection and factors associated with thinness and overweight/obesity. Cad Saude Publica 2011; 27(10):1997-2008.

65. Koethe JR, Heimburger DC, PrayGod G, Filteau S. From Wasting to Obesity: The Contribution of Nutritional Status to Immune Activation in HIV Infection. J Infect Dis 2016; 214(Supl. 2):S75-S82.
66. Tiozzo E, Konefal J, Adwan S, Martinez LA, Villabona J, Lopez J, Cutrono S, Mehdi S, Rodriguez A, Woolger JM, Lewis JE. A cross-sectional assessment of metabolic syndrome in HIV-infected people of low socio-economic status receiving antiretroviral therapy. Diabetol Metab Syndr 2015; 7(1):15.

67. Rasmussen LD, Mathiesen ER, Kronborg G, Pedersen C, Gerstoft J, Obel N. Risk of Diabetes Mellitus in Persons with and without HIV: A Danish Nationwide Population-Based Cohort Study. PLoS ONE 2012; 7(9):e44575.

68. Tripathi A, Liese AD, Jerrell JM, Zhang J, Rizvi AA, Albrecht H, Duffus WA. Incidence of diabetes mellitus in a population-based cohort of HIV-infected and non-HIV-infected persons: the impact of clinical and therapeutic factors over time. Diabet Med 2014; 31(10):1185-1193.

69. Lazzaretti RK, Gasparotto AS, Sassi MGDM, Polanczyk CA, Kuhmmer R, Silveira JM, Basso RP, Pinheiro CAT, Silveira MF, Sprinz E, Mattevi VS. Genetic Markers Associated to Dyslipidemia in HIV-Infected Individuals on HAART. TSWJ 2013; 2013:1-10.

70. Puttawong S, Prasithsirikul W, Vadcharavivad S. Prevalence of lipodystrophy in Thai-HIV infected patients. J Med Assoc Thai 2004; 87(6):605

71. Polsky S, Floris-Moore M, Schoenbaum E, Klein R, Arnsten J, Howard A. Incident hyperglycaemia among older adults with or at-risk for HIV infection. Antivir Ther 2011; 16(2):181-188.

72. Galli L, Salpietro S, Pellicciotta G, Galliani A, Piatti P, Hasson H, Guffanti M, Gianotti N, Bigoloni A, Lazzarin A, Castagna A. Risk of type 2 diabetes among HIV -infected and healthy subjects in Italy. Eur J Epidemiol 2012; 27(8):657-665.

Artigo apresentado em 08/02/2018

Aprovado em 05/07/2018

Versão final apresentada em 07/07/2018 